\title{
Paradigmenwechsel in der Ernährungstherapie metabolischer Erkrankungen?
}

Kalorienreduzierte Diätformen sind im wahrsten Sinne des Wortes in aller Munde - und das nicht erst seit Kurzem: Bereits 1869 löste William Banting eine ganz Europa erfassende Diätwelle mit seiner deutlich kohlenhydratarmen Kost aus. Fast einhundert Jahre später, in den 70er-jahren des letzten Jahrhunderts, wurde dieses Konzept von Robert Atkins aufgegriffen und durch eine enorme Medienpräsenz erneut populär gemacht (wobei die wesent-

\section{Die Hinweise mehren} sich: Low Carb als therapeutische Kostform liche Modifikation der Verzicht auf die bei Banting erheblichen Alkoholmengen war). Wissenschaftlich war die Atkins-Diät bis vor wenigen Jahren durchaus verpönt und nicht akzeptiert und zwar wegen mutmaßlich ungünstiger Wirkungen auf den Cholesterinstoffwechsel. Anfang dieses Jahrtausends lieferten dann aber mehrere kontrollierte Studien den Nachweis, dass kohlenhydratreduzierte Diätformen nicht nur wirksam und unbedenklich, sondern sogar günstig hinsichtlich kardiovaskulärer Risikofaktoren des Fett- und Glukosestoffwechsels sind. Das lange gepflegte Vorurteil gegen diese Diätformen und die Ansicht, eine fett- und eiweißreiche Kost wäre der Auslöser von Herz-Kreislauf-Erkrankungen und Diabetes, wurden damit im Mark erschüttert.

Parallel zur begründbaren Akzeptanz kohlenhydratarmer Diäten gewannen in den letzten Jahren die Begriffe glykämischer Index (Gl), glykämische Last oder auch glykämisches Glukoseäquivalent zunehmend Raum in der Fachpresse, weit mehr aber in der Laienpresse. Trotz oder gerade wegen der großen Schwierigkeiten, aus den glykämischen Indices einzelner Nahrungsmittel eine ganze Kostform mit ebenfalls bekanntem Gl zu entwickeln, suggerierte der Begriff des Gl eine Wissenschaftlichkeit, die sich nur schwer be- legen ließ. Wie es um den Stellenwert des GI und der davon abgeleiteten Begriffe bestellt ist, schildert in diesem Heft Hans-Joachim F. Zunft in seinem Übersichtsartikel "Glykämischer Index und Glykämische Last - theoretisches Konstrukt oder überzeugendes Beratungskonzept?".

Den praktischen Aspekt der Wirksamkeit kohlenhydratarmer Diätformen beschreibt zudem Peter Heilmeyer in seinen Untersuchungen zur LOGI-Methode. Interessanterweise stand LOGI ursprünglich für Low Glycemic Index und wurde später als Low Glycemic and Insulinemic Diet umgedeutet. Die neue Begrifflichkeit beschreibt eine Kostform, die postprandiale Blutzucker- und Insulinspitzen verhindern hilft. Dies gelingt praktisch durch eine Senkung der Kohlenhydratmenge. Ein wesentlicher, wenn nicht sogar der wichtigste Effekt dieser Kostform, ist die automatische Reduktion der aufgenommenen Kalorienmenge, vermittelt wahrscheinlich durch ein anhaltenderes Sättigungsgefühl. Dies wurde auch in kontrollierten Studien zu kohlenhydratarmen Diäten oder der Atkins-Diät gefunden.

Klar ist, dass - nach der Bewegungsarmut - der Kaloriengehalt der Nahrung die wichtigste Ursache der Adipositas- und Diabetes-Epidemie ist. Die Hinweise mehren sich dabei, dass eher dem Kohlenhydratanteil in der Nahrung als dem Fett- oder Eiweißanteil die Hauptrolle als letztlich dickmachender Energieträger zukommt.

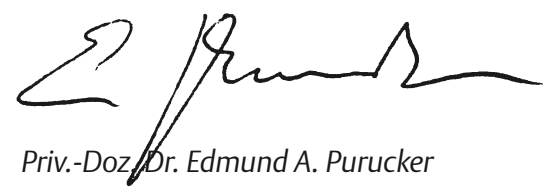

